\title{
Modafinil and $\mathrm{y}$-hydroxybutyrate have sleep state-specific pharmacological actions on hypocretin-1 physiology in a primate model of human sleep
}

\author{
Jamie M. Zeitzer, PhD ${ }^{1,2}$, Christine L. Buckmaster, BS ${ }^{1}$, Hans-Peter Landolt, PhD ${ }^{3}$, David M. \\ Lyons, $\mathrm{PhD}^{1}$, and Emmanuel Mignot, $\mathrm{MD}, \mathrm{PhD}^{1,4}$ \\ ${ }^{1}$ Department of Psychiatry and Behavioral Sciences, Stanford University; Stanford CA, USA 2 \\ Psychiatry Service, VA Palo Alto Health Care System; Palo Alto CA, USA ${ }^{3}$ Institute of Pharmacology \\ \& Toxicology and Zürich Center for Integrative Human Physiology, University of Zürich; Zürich, \\ Switzerland ${ }^{4}$ Howard Hughes Medical Institute, Stanford University; Stanford CA, USA
}

\begin{abstract}
Hypocretin-1 is a hypothalamic neuropeptide that is important in the regulation of wake and the lack of which results in the sleep disorder narcolepsy. Using a monkey that has consolidated wake akin to humans, we examined pharmacological manipulation of sleep and wake and its effects on hypocretin physiology. Monkeys were given the sleep-inducing gamma-hydroxybutyrate (GHB) and the wake-inducing modafinil both in the morning and in the evening. Cerebrospinal fluid (CSF) hypocretin- 1 concentrations changed significantly in response to the drugs only when accompanied by a behavioral change (GHB-induced sleep in the morning or modafinil-induced wake in the evening). We also found that there was a large (180-fold) inter-individual variation in GHB pharmacokinetics that explains variability in sleep-induction in response to the drug. Our data indicate that the neurochemical concomitants of sleep and wake are capable of changing the physiological output of hypocretin neurons. Sleep independent of circadian timing is capable of decreasing CSF hypocretin-1 concentrations. Furthermore, hypocretin neurons do not appear to respond to an "effort" to remain awake, but rather keep track of time spent awake as a wake-promoting counterbalance to extended wakefulness.
\end{abstract}

\section{Keywords}

orexin; hypocretin; sleep; pharmacology; modafinil; gamma-hydroxybutyrate

\section{Introduction}

Hypocretin- 1 is a neuropeptide produced in the lateral hypothalamus. Although it is likely involved in the regulation of multiple behaviors (Berridge \& España 2005), a major function across species may be the maintenance of extended wake, most notably in wake-consolidated mammals (Zeitzer \& Mignot 2006). The release of hypocretin-1 is partially controlled by the circadian clock (Deboer et al. 2004;Zhang et al. 2004), with elevated release normally occurring late in the wake period in both nocturnal (Yoshida et al. 2001) and diurnal (Zeitzer

Corresponding author: Jamie M. Zeitzer, 3801 Miranda Avenue (151Y), Palo Alto CA 94304 USA. Telephone: 650-493-5000 extension 62410; telefax: 650-852-3297; jzeitzer@stanford.edu.

Disclosures/Conflicts of Interest: A portion of this study was funded by Jazz Pharmaceuticals (JMZ). 
et al. 2003) mammals. Hypocretin is wake promoting when injected into the central nervous system (Hagan et al. 1999; Yoshimichi et al. 2001) and increased wake transitions occur following optogenic stimulation of hypocretin neurons in vivo (Adamantidis et al. 2007). Forced wake can also cause an increase in hypocretin-1 concentrations in hypothalamic dialysate (Yoshida et al. 2001) or cerebrospinal fluid (CSF) (Deboer et al. 2004;Zeitzer $e t$ al. 2007). It is hypothesized that this increase contributes to the actual extension of wake, opposing the mounting sleep debt. Similarly, hypocretin decreases upon sleep onset, with minimal levels late in the inactive period, possibly helping to maintain sleep in the face of reduced sleep debt. Problematically however, it is not known whether the increased hypocretin levels that have been reported with sleep deprivation are a reflection of the need for higher hypocretin tone to further stimulate wake, or may simply be invoked by the sleep deprivation procedure and its behavioral correlates. It is also unknown if the decline in CSF hypocretin-1 concentrations that is observed during the time of normal sleep is due to sleep itself or a circadian signal.

The study of hypocretin-1 physiology is difficult in humans because significant concentrations of this peptide are only found in CSF and brain tissue. Lumbar CSF concentrations of hypocretin-1 exhibit a diurnal rhythm (Salomon et al. 2003), but the amplitude is quite small and the rhythm is likely delayed by several hours due to the transit time between brain release and equilibration in the lumbar sac. Most animal models (e.g., rats, cats, dogs, mice) of hypocretin-1 physiology are also problematic because the relationship between sleep and circadian rhythms is fundamentally different in humans compared to most other mammals. Human sleep typically occurs as a single daily episode, while in most other mammals sleep is polyphasic, in that it occurs in brief bouts throughout the day and night with grouping of the bouts during daytime (diurnal), night time (nocturnal), or at dawn/dusk (crepuscular). Given that hypocretin is likely involved in the maintenance of consolidated daytime wake in humans, it is critical to examine its physiology in an animal model that has an equivalent maintenance of wake. As such, we have developed a primate model to study the normal physiology of hypocretin-1 - the squirrel monkey (Zeitzer, et al. 2003, 2004,. 2007). These New World primates have consolidated sleep and wake akin to humans, unlike most other mammals, and CSF is readily accessible via a percutaneous tap of the cerebellomedullary cistern (cisterna magna).

In this study, we tested the hypothesis that sleep itself can cause a decrease in CSF hypocretin-1 concentrations. Furthermore, we investigated whether the increase in CSF hypocretin-1 concentrations in response to sleep deprivation (Zeitzer et al. 2007; Deboer et al. 2004; Yoshida et al. 2001) occurs due to an extension of wake or whether this increase only reflects a compensatory effort to remain awake when forced to do so by an experimental protocol (e.g., secondary to cortisol or other neurochemical changes). To induce sleep, we used sodium oxybate, also called gamma-hydroxybutyrate (GHB). This compound is known to greatly increase slow wave sleep (Crunelli, which is in contrast to most other currently used hypnotics such as benzodiazepines. GHB is typically used at night (bi-nightly administration) to consolidate sleep in hypocretin-deficient humans with narcolepsy, an effect that subsequently improves daytime wake (and other symptoms) in these individuals. To produce wake, we used modafinil, a stimulant that appears to have fewer unwanted effects than other stimulant compounds and does not strongly affect sleep state distribution in subsequent sleep (Shelton et al. 1995). Modafinil is a common wake-promoting agent used in the treatment of narcolepsy and other conditions associated with excessive daytime sleepiness. Of interest, there have been reports linking the mechanism of action modafinil to activation of hypocretin-producing neurons (Chemelli et al. 1999; Scammell et al. 2000). As controls, we also administered GHB at night, during which time it would be expected to have no effect on the amount of sleep observed, and we administered modafinil during the day during which time it would be expected to have no effect on the amount of wake observed. We also have examined changes 
in plasma cortisol as we wanted to ensure that changes we observed were not due to the nonspecific stress of maintaining wake, as occurs in every study of behavioral sleep deprivation (e.g., gentle handling, forced locomotion) in non-human mammals. These controls allow us to discriminate the direct effects of the drugs per se and the indirect effects of the drugs mediated through changes in sleep and wake.

\section{Methods}

\section{Subjects}

Six adult, male squirrel monkeys (Saimiri sciureus sciureus) from the Stanford University squirrel monkey colony were examined in this study. Monkeys were group housed in two cages in the same room under constant humidity and temperature, with a $12 \mathrm{~h}: 12 \mathrm{~h}$ light:dark cycle (room lights on at 07:00, room lights off at 19:00). Dim yellow lighting was always on during hours of scheduled darkness, which yellow light does not interfere with circadian entrainment in these monkeys (Zeitzer and Buckmaster, unpublished observations). Food (New World Primate Diet 5040, PMI Nutrition International, Brentwood MO; fresh fruits and vegetables) was freely available and replenished daily at 10:00, except on experimental days, when this was done at 13:00. All husbandry and experimental procedures were reviewed and approved by the Stanford University Administrative Panel on Laboratory Animal Care and done in accordance with the Guide for the Care and Use of Laboratory Animals as adopted and promulgated by the U.S. National Institutes of Health.

\section{General Protocol}

Each monkey had CSF and blood obtained on six separate occasions, with at least two weeks between each occasion. Three samples were obtained at 13:00- one after placebo administration, one after GHB administration, and one after modafinil administration. The other three samples were obtained at 01:00 - one after placebo injections, one after GHB administration, and one after modafinil administration. All samples were assayed in duplicate for CSF hypocretin-1 and cortisol. Blood samples obtained after GHB administration were assayed for GHB concentrations and those obtained after modafinil administration were assayed for modafinil concentrations. Activity patterns were monitored throughout the protocol; in prior studies, these have been shown to accurately reflect sleep (Zeitzer $e t$ al. 2003).

\section{Drug Administration}

In preliminary testing, based on doses used to treat humans with narcolepsy and concentrations administered in behavioral studies of rhesus monkeys (Woolverton et al. 1999; Nakamura et al. 1987;Beardsley et al. 1996; Snead III 1978), we determined the dose of GHB needed to induce sleep in monkeys from 07:00-13:00 (confirmed by visual observation). In the Xyrem formulation (Jazz Pharmaceuticals, CA), GHB is in suspension at $500 \mathrm{mg} \cdot \mathrm{ml}^{-1}$. Though administered orally in humans, we were unable to orally administer GHB in this formulation because the monkeys refused to ingest GHB that had been mixed with any of their favorite treats (e.g., marshmallows, peanut butter, jelly). We, therefore, administered GHB in the Xyrem formulation using intramuscular injections into the hip flexor. A single dose of GHB did not induce continuous sleep from 07:00-13:00, likely due to the short half-life of the compound (0.5-1 h in humans). Therefore, we administered $200 \mathrm{mg} \cdot \mathrm{kg}^{-1}$ of GHB at 06:00 and $175 \mathrm{mg} \cdot \mathrm{kg}^{-1}$ of GHB at 10:00 (for CSF and blood collection at 13:00) and $200 \mathrm{mg} \cdot \mathrm{kg}^{-1}$ of GHB at 18:00 and $175 \mathrm{mg} \cdot \mathrm{kg}^{-1}$ of GHB at 22:00 (for CSF and blood collection at 01:00). This dosing schedule did not induce significant side effects (e.g., nausea and ataxia).

In preliminary testing, based on doses typically administered to human children and administered to rhesus monkeys and marmosets in behavioral studies (Herman et al. 
1991;Lagarde \& Milhaud 1990;Ivanenko et al. 2003; van Vliet et al. 2008), we determined the dose of modafinil needed to keep monkeys awake from 19:00-01:00. We found that 16 $\mathrm{mg} \cdot \mathrm{kg}^{-1}$ of modafinil kept the monkeys awake for three hours and repeated dosing could keep them awake for six hours. We packaged $16 \mathrm{mg} \cdot \mathrm{kg}^{-1}$ of modafinil (Lafon, France) into gelatin capsules and placed the capsules inside a miniature marshmallow, a favorite treat of our squirrel monkeys. The marshmallows-capsules were given to the monkeys at 07:00 and 10:00 (for CSF and blood collection at 13:00) or at 19:00 and 22:00 (for CSF and blood collection at 01:00).

In the third condition (placebo), we administered $0.4 \mathrm{ml}$ sterile water into the hip flexor at 07:00 and 10:00 (for CSF and blood collection at 13:00) or at 19:00 and 22:00 (for CSF and blood collection at 01:00).

\section{Activity monitoring}

All monkeys wore a collar-mounted actigraph (Actiwatch-64, MiniMitter, Bend OR), a small $(17 \mathrm{~g})$ device capable of detecting movement in three dimensions through the use of an accelerometer. Data were stored as integrated (total) movement (arbitrary units) occurring over one minute intervals. Actigraphs were worn throughout the protocol and at least one week of adaptation occurred before the collection of analyzable data. Sleep and wake were estimated from movement data using Sleepwatch software (v.2.82, Cambridge Neurotechnology, Cambridge UK) set on low sensitivity, which has been shown to accurately reflect sleep and wake in squirrel monkeys (Zeitzer et al. 2003). Data were integrated using the trapezoidal method from 19:00-01:00 (for CSF collection at 01:00) or 07:00-13:00 (for CSF collection at 13:00). Actigraphy was unsuccessful in 3 of 36 collection intervals (one 13:00 baseline collection, one 13:00 modafinil collection, one 01:00 modafinil collection).

\section{CSF and Blood Collection}

CSF was obtained via a percutaneous tap from the cisterna magna (cerebellomedullary cistern) from anesthetized monkeys using a siliconized syringe (Zeitzer et al. 2003). Anesthesia was induced by intrasaphenous injection of $10.0 \mathrm{mg} \cdot \mathrm{kg}^{-1}$ ketamine hydrochloride with 0.5 $\mathrm{mg} \cdot \mathrm{kg}^{-1}$ diazepam, and supplemented as needed with an intramuscular injection of 5.0 $\mathrm{mg} \cdot \mathrm{kg}^{-1} \mathrm{ketamine}$ hydrochloride. CSF (200 $\left.\mu \mathrm{l}\right)$ was collected, placed in a siliconized tube on ice, and later stored at $-80^{\circ} \mathrm{C}$. Blood was obtained via puncture of the femoral vein. Blood was spun in a centrifuge $\left(4900 \mathrm{rpm}, 4^{\circ} \mathrm{C}, 14 \mathrm{~min}\right)$ with the resulting supernatant being collected and stored at $-80^{\circ} \mathrm{C}$.

\section{Assays}

Blood concentrations of GHB were determined by Covance Laboratories (Madison WI; Report \#8022-100) using liquid chromatography with tandem mass spectrometric detection. Blood concentrations of modafinil were determined using liquid chromatography - mass spectrometry (limit of detection: $0.7 \mathrm{ng} \cdot \mathrm{ml}^{-1}$; limit of quantification: $2.4 \mathrm{ng} \cdot \mathrm{ml}^{-1}$; signal-to-noise ratio: 3 and 10, respectively) (Bodenmann et al., submitted). Hypocretin-1 concentrations were determined using a commercially available radioimmunoassay kit and a custom hypocretin-1 antibody, provided by Drs. Shahrad Taheri and Ling Lin (detection limit $=10 \mathrm{pg} \cdot \mathrm{ml}^{-1}, 5 \%$ intra-assay variability, inter-assay variability adjusted with internal standard, Phoenix Pharmaceuticals, Belmont CA) (Zeitzer et al. 2003). We selected the two CSF sampling time points of 13:00 and 01:00 because our previous research (Zeitzer et al. 2003) showed that CSF hypocretin-1 values are intermediate at these times and, thus, could be observed to increase or decrease without a concern of a ceiling or floor effect. Plasma concentrations of cortisol were determined using a commercially available radioimmunoassay (Diagnostic Products Inc., Los Angeles CA). All samples were assayed in duplicate. 


\section{Statistics}

Student's $t$-tests and basic calculations were performed using Microsoft Excel (Redmond WA; v. 11.8211.8202). Curve fitting of concentration-response data was performed using Microcal Origin (Northampton MA; v. 6.1052). Repeated measure two-factor analyses of variance (ANOVA) and Spearman Rank correlation analyses were performed using SAS Statview (Cary NC; v. 5.0.1). To determine significance of change in the amount of movement or wake, data were compared as change from time-matched baseline to respective drug condition. The absorbance and body distribution of some drugs are affected by either body mass index (BMI) or body surface area (BSA). BMI was calculated as $\mathrm{kg} \cdot \mathrm{m}^{-2}$. Height was measured as the crownrump length and this distance was used in the BMI calculation. BMI calculated with crownrump length had a better correlation with plasma leptin levels, a fat cell-produced hormone known to correlate with BMI in humans, than when other measures of height were used to calculate BMI (Zeitzer et al. 2007). BSA was calculated as $(\mathrm{cm} \cdot \mathrm{kg} \cdot 0.0002778)^{0.5}$ (Mosteller 1987). All data are presented as mean \pm standard error of the mean.

\section{Results}

As expected, morning administration of the sleep-promoting compound GHB increased the amount of sleep while administration of the wake-promoting compound modafinil had negligible effects on wake during the daytime. After morning administration of GHB, there was a significant $57 \pm 17 \%$ decline in the amount of movement, as compared with the control condition ( $p<0.05$, paired $t$-test) (Figure 1). In deriving estimated sleep/wake from actigraphy data, there was also a corresponding significant $65 \pm 11 \%$ decrease in the amount of wake after morning administration of GHB compared to the control condition ( $p<0.01$, paired $t$-test). Confirming previous reports (Wisor et al. 2001), after morning administration of modafinil, there was no significant change in the amount of movement occurring 07:00-13:00, as compared with the control condition ( $p=0.83$, paired $t$-test) (Figure 1$)$. There was also no significant change in the amount of wake after exposure to modafinil as compared to the control condition ( $p=0.12$, paired $t$-test). Thus, morning administration of GHB significantly decreased the amount of wake while morning administration of modafinil did not significantly change the amount of activity or wake.

As expected and in contrast to daytime administration, evening administration of the same dose of modafinil increased the amount of wake and administration of the sleep-promoting GHB had negligible effects on sleep. After evening administration of GHB, there was a nonsignificant decrease $(-22 \pm 4 \%)$ in the amount of movement, as compared with the control condition ( $p=0.07$, paired $t$-test) (Figure 2 ). There was also no significant change in the amount of wake $(-15 \pm 19 \%)$ after evening administration of GHB, compared with the control conditions $(p=0.09$, paired $t$-test). After evening administration of modafinil, there was a nonsignificant increase $(410 \pm 272 \%)$ in the integrated amount of movement (20:00-01:00), as compared with the control conditions ( $p=0.16$, paired $t$-test) (Figure 2$)$. In deriving estimated sleep/wake from actigraphy data, there was, however, a significant $859 \pm 525 \%$ increase in the amount of wake after exposure to modafinil as compared to control conditions $(p<0.01$, paired $t$-test). Thus, evening administration of modafinil significantly increased the amount of wake, while evening administration of GHB had no significant influence on the amount of wake.

We next examined the relationship between plasma concentrations of GHB and changes in wake. Surprisingly, we found a 180 -fold difference in plasma GHB concentrations, ranging $0.521-95.0 \mu \mathrm{g} \cdot \mathrm{ml}^{-1}$. This was not due to inaccurate experimental procedure as rank ordered circulating concentrations of GHB after daytime or nighttime administration correlated highly within monkeys ( $\mathrm{r}=0.94, p<0.05$, Spearman rank correlation coefficient). There existed a significant concentration-effect relationship between morning administration of GHB and wake between 07:00 and 13:00, such that a logistic function adequately described the data 
$\left(\mathrm{R}^{2}=0.80, p<0.01\right.$ ) (Figure 3), suggesting that only concentrations above $\sim 10 \mu \mathrm{g} \cdot \mathrm{ml}^{-1}$ were pharmacologically active at inducing sleep during the daytime. This relationship was maintained when only the GHB dosing, and not the placebo dosing, was used in the model $\left(\mathrm{R}^{2}=0.68, p<0.05\right)$. No such relationship existed between nighttime administration of GHB and wake between 19:00 and 01:00 (during the night, logistic function: $\mathrm{R}^{2}=0.23, p>0.09$; linear function: $\mathrm{R}^{2}=0.12, p=0.27$ ), when the monkeys were already mostly asleep. The GHB was dosed on a mg of drug per kg of monkey basis. Retrospective analyses of GHB rank order (averaged morning and evening data) indicated no significant relationship between circulating GHB concentrations and weight ( $\mathrm{r}=-0.20, p=0.70$ Pearson correlation), BMI ( $\mathrm{r}=0.52, p=0.29$ Pearson correlation), or BSA ( $\mathrm{r}=0.06, p=0.91$ Pearson correlation), suggesting other intrinsic differences are the cause of the wide spread of plasma GHB concentrations.

Unlike GHB, there was only a 0.4-fold difference between individual animals in plasma modafinil concentrations, $12.25-17.67 \mu \mathrm{g} \cdot \mathrm{ml}^{-1}$. Furthermore, rank orders of modafinil concentrations were not similar between the two dose times ( $\mathrm{r}=0.46, p=0.36$, Spearman rank correlation coefficient), indicating that the minor variation in circulating modafinil was likely due to slight difference in experimental procedure or in assessment of drug plasma concentrations. As expected, since modafinil was dosed to be equivalent based on body weight, there were no significant correlations between plasma modafinil concentrations and weight $(\mathrm{r}=$ $-0.79, p=0.06)$, BMI $(\mathrm{r}=0.00, p=1.00)$, or BSA $(\mathrm{r}=-0.41, p=0.41)$.

ANOVA indicated that there was a significant effect of both time of day $(p<0.05)$ and drug condition $(p<0.01)$ on CSF concentrations of hypocretin-1 (Figure 4). Post-hoc analyses with paired $t$-tests showed that CSF hypocretin- 1 concentrations were significantly lower after GHB administration during the daytime $(795.6 \pm 42.9 \mathrm{pg} / \mathrm{mL}$ vs. $640.0 \pm 35.2 \mathrm{pg} / \mathrm{mL}$, baseline vs. $\mathrm{GHB}, p<0.05)$ when sleep-inducing effects occurred, but not during the night $(618.6 \pm 42.3$ $\mathrm{pg} / \mathrm{mL}$ vs. $585.9 \pm 26.3 \mathrm{pg} / \mathrm{mL}$, baseline vs. GHB, $p=0.51$ ) when sleep-inducing effects were masked by natural sleep. We found no relationship between the percentage change in GHBinduced sleep at 13:00 and the percentage change in hypocretin- 1 concentrations $(r=0.52$, $p=0.37$ Pearson correlation; $\mathrm{r}=0.74, p=0.09$ logistic function). After daytime modafinil administration, there was a non-significant increase in CSF hypocretin- 1 concentrations, to $917.6 \pm 51.7 \mathrm{pg} / \mathrm{mL}(p=0.18)$, but the changes after administration at night to $738.1 \pm 35.1 \mathrm{pg} /$ $\mathrm{mL}$ were significant $(p<0.05)$. Unexpectedly, we found that the larger the percentage change in modafinil-induced wake at 01:00, the smaller the percentage change in hypocretin-1 concentration ( $\mathrm{r}=0.87, p<0.01$ Pearson correlation; $\mathrm{r}=0.99, p<0.01$ logistic function).

As with hypocretin-1, we also examined plasma cortisol concentrations at 13:00 and 01:00. At 13:00, cortisol concentrations were 177.3 $\pm 73.7 \mu \mathrm{g} / \mathrm{dL}$ (placebo), $180.1 \pm 44.9 \mu \mathrm{g} / \mathrm{dL}$ (GHB), and 252.2 \pm 35.3 (modafinil), while at 01:00 they were $96.6 \pm 48.0 \mu \mathrm{g} / \mathrm{dL}$ (placebo), $87.6 \pm$ $20.9 \mu \mathrm{g} / \mathrm{dL}$ (GHB), and $152.7 \pm 62.7 \mu \mathrm{g} / \mathrm{dL}$ (modafinil), showing the expected diurnal variation. There was a significant effect of time of day $(p<0.01)$ and of drug administration $(p<0.02)$ (Figure 5). There were no significant differences in plasma cortisol following GHB administration during the daytime $(p=0.94)$ or at night $(p=0.63)$. Post - hoc analyses using paired $t$-tests indicated that there was a significant increase in plasma cortisol concentrations following modafinil administration during the daytime $(p<0.01)$, but not at night $(p=0.25)$.

\section{Discussion}

Using an animal that has consolidated sleep and wake, we examined the impact of sleep and wake on hypocretin regulation through their pharmacological induction with GHB and modafinil. We found that CSF hypocretin- 1 concentrations declined after exposure to the sleeppromoting GHB and increased after exposure to the wake-promoting modafinil. These effects, however, are not due to the direct action of these drugs on hypocretin activity, as the effects 
were only present when there was a concomitant change in behavior (i.e., an increase or a decrease in sleep amounts, respectively). Thus, the neurochemical concomitants of sleep and wake, rather than the direct effects of the drugs, are capable of changing the physiological output of hypocretin neurons.

Sleep induced by GHB lowers CSF hypocretin-1 concentrations. GHB in and of itself, however, does not have a significant effect on CSF hypocretin-1 concentrations. By the nature of the model, previous studies in polyphasic mammals have been unable to establish whether the decline of CSF hypocretin-1 concentrations during the sleep portion of the daily cycle is due to a sleep or time of day effect. Our data indicate that sleep is independently able to reduce CSF hypocretin-1 concentrations. This conforms well to electrophysiological recordings of hypocretin neurons in rodents that show modest decrease in firing of hypocretin neurons during times of sleep (Mileykovskiy et al. 2005;Lee et al. 2005). While our data are limited, in that we did not record electroencephalic activity to confirm sleep, we have demonstrated previously that behavioral quiescence during wake does not induce changes in CSF hypocretin-1 concentrations (Zeitzer et al. 2004), thus confirming that the GHB-induced changes we observed are mediated through sleep and are not due to a reduction in overall activity. The relative contribution of sleep, specific phases of sleep, specific electroencephalographic characteristics of sleep, and the circadian clock, however, remain to be elucidated.

GHB is an endogenous metabolite of GABA (Doherty et al. 1978) that can be converted to GABA via transamination. There are specific GHB receptors in the squirrel monkey brain (Castelli et al. 2000), but it is likely that most if not all of the soporific actions of GHB are mediated by GABA-B receptors (Crunelli et al. 2006;Carai et al. 2008). Our data showed that there were extremely large variations in circulating GHB concentrations despite the monkeys each being dosed with the same number of $\mathrm{mg}$ of drug per $\mathrm{kg}$ body weight. The dose was always given as an injection and the monkeys were injected in a random order. This variation appeared to be a trait of the monkeys, as the rank order of concentrations was similar at both dosing times. Importantly, this variation was functionally significant, as circulating concentrations of GHB predicted the amount of drug-induced sleep that the monkey experienced. We examined if the variation could be due to common predictors of drug concentrations in plasma, such as weight, body mass index, and body surface area, but none of these explained the variance we observed. We could find no published studies that have described variation in GHB pharmacokinetics. It is possible that inter-individual variation in absorption of the GHB suspension may account for some of the inter-individual variation in plasma concentrations of GHB. While this finding may be idiosyncratic for the squirrel monkey, further examination of GHB pharmacokinetics in this animal model is warranted.

We demonstrate here that an extension of wake can increase CSF hypocretin- 1 concentrations and that this increase is not dependent upon the monkeys being forced to maintain wake through interactions with human observers. We have shown previously in squirrel monkeys that forcing the monkeys to remain awake for up to 7 hours past their normal wake time, by gently shaking the cage or perch on which the monkey is resting, is sufficient to maintain hypocretin-1 concentrations at an elevated value (Zeitzer et al. 2007). Others have shown a similar phenomenon in other species (Yoshida et al. 2001;Deboer et al. 2004;Wu et al. 2002). None of these non-human mammals stay awake volitionally as can humans. To keep a rat or a monkey awake, it must be provided with external motivation. Thus, the effects on hypocretin-1 physiology could be secondary to physiologic changes that occur in response to this motivation. We have previously shown and confirmed here that cortisol does not have a significant contribution to the hypocretin-1 signal in squirrel monkeys (Zeitzer et al. 2007), but there may be other factors that do contribute. In this study, after monkeys were administered modafinil, they were left undisturbed until they were gathered for CSF sampling. Due to the pharmacological properties of modafinil, they were able to maintain wake for much of this 
time. Therefore, it is unlikely that the monkeys were expending any "effort" to remain awake nor were they stressed into a state of wakefulness, as indicated by the lack of a change in plasma cortisol. Despite this, hypocretin-1 concentrations were elevated over control conditions. This implies that hypocretin-1 changes during sleep deprivation are not due to the motivated actions of the monkey to remain awake or to changes in behavior induced by physical intervention, but are primarily due to being awake for an extended length of time. The changes in hypocretin-1 concentrations are likely not directly due to the action of the drug, as when modafinil was administered during the daytime, there was no significant, consequent effect on CSF hypocretin-1 concentrations. In this manner, hypocretin- 1 appears to act more as a measure of the length of time awake then it does as a reactive mechanism to offset sleepiness. This result is also supported by the data of Rao and colleagues (Rao et al. 2007), who demonstrated that prolonged wake in mice, induced by gentle handling or modafinil, increases presynaptic glutamatergic input to hypocretin neurons. As reported for other glutamatergic systems in the brain (Tononi \& Cirelli 2007), hypocretin neurons may thus increase activity with extended wake, resulting in long term potentiation. In the process, hypocretin neurons, therefore, may be acting as a counter for daily wake until the process becomes somehow unsustainable, then reducing activity and inducing sleep.

There have been many theories as to the mechanism of action of modafinil, including activation of hypocretin neurons, but there is no consensus agreement. Its main target for the promotion of wake is likely to be the dopamine transporter (Wisor et al. 2001), potentially acting through downstream dopaminergic (Qu et al. 2008) or noradrenergic mechanisms (Wisor \& Eriksson 2005). Modafinil does selectively increase Fos expression in mouse (Chemelli et al. 1999) and rat (Scammell et al. 2000) hypocretin neurons and we did observe an increase in CSF hypocretin-1 during the daytime after modafinil administration, but this did not reach the level of statistical significance. Our results suggest, rather, that modafinil-induced increases in Fos expression in rodent hypocretin neurons are indirectly due to a change in wake and not due to a direct action of the drug. The observation that bath-applied modafinil does not change the firing activity of hypocretin neurons in murine hypothalamic slices (Rao et al. 2007) also supports this hypothesis. Also, importantly, hypocretin does not appear necessary for the wakepromoting actions of modafinil, as mice genetically constructed to not produce hypocretins have a robust modafinil-induced wake-response (Williel et al. 2005). Further confirmation with electroencephalographic recording of sleep will be important.

Another unexpected finding was the possible state-dependent effect of modafinil on cortisol. While GHB had no effects on cortisol, modafinil increased cortisol concentrations when the drug was administered in the morning, when there were no obvious behavioral changes after modafinil administration, but not in the evening, when the monkeys experienced a significant increase in their amount of wake. There have been few studies on the effect of modafinil on cortisol (the main stress-related hormone primates) or corticosterone (the main stress-related hormone in rodents). One study in humans reported that modafinil (300 mg, $4.5 \mathrm{mg} \cdot \mathrm{kg}^{-1}$ for a $66 \mathrm{~kg}$ human) had no effect on plasma cortisol concentrations (Brun et al. 1998). Another study in mice reported that $16 \mathrm{mg} \cdot \mathrm{kg}^{-1}$ or $32 \mathrm{mg} \cdot \mathrm{kg}^{-1}$ did not affect plasma corticosterone concentrations (Béracochéa et al. 2008), but the same group reported in a separate study that both $16 \mathrm{mg} \cdot \mathrm{kg}^{-1}$ and $32 \mathrm{mg} \cdot \mathrm{kg}^{-1}$ of modafinil increased plasma corticosterone concentrations, while $8 \mathrm{mg} \cdot \mathrm{kg}^{-1}$ of modafinil did not (Piérard et al. 2006). Both of these studies in mice examined corticosterone during the normal time of sleep in mice (daytime) and we only saw effects during the normal time of wake in monkeys (daytime) in our study. Given the lower dose of modafinil used in the one human study, it is possible that only at higher doses and during time of normal wake are the effects of modafinil on cortisol observed. More research is needed to explore the possible state-dependent effects of modafinil on plasma cortisol. 
This study also has implication for diagnostic studies of CSF in human narcolepsy and for the interpretation of pharmacology studies that should examine sleep as a confounder. The fact that the same compound administered at the same dose at two different times of the day has strikingly differential effects is important, as it suggests that studies looking at the neurochemical effects of various drugs must control not only for circadian time but also for sleep history when studying effects on neurotransmitters known to be involved in sleep regulation. CSF hypocretin-1 concentration is often used as a diagnostic tool in sleep medicine, notably to confirm unusual presentations of narcolepsy (Mignot et al. 2002). Our findings suggest that if patients were using either GHB (typically given at night) or modafinil (typically given during the daytime), it is unlikely that these would have a significant impact on the clinical utility of CSF hypocretin-1 measurement, as changes these drugs evoked in hypocretin-1 concentrations via modulation of sleep and wake activity would likely be minimal in comparison to diagnostic criteria $(<110 \mathrm{pg} / \mathrm{mL}$ are indicative of narcolepsy and $>200 \mathrm{pg} /$ $\mathrm{mL}$ is normal).

We have shown in this study that sleep is independently capable of causing a decrease in CSF hypocretin-1 concentrations, and that an extension of wake, rather than efforts related to maintaining wake, is sufficient to cause an increase in CSF hypocretin- 1 concentrations. Determination of the upstream pathways that control the effects of sleep and wake on hypocretin neuron activity will be crucial to better understand the physiology of this system.

\section{Acknowledgments}

The authors would like to thank Dr. Seiji Nishino for providing the modafinil, S. Bodenmann and Dr. M. Arand for help with modafinil quantification, and Dr. William Houghton for early advice on GHB dosing.

Funding: This work was funded by NARSAD (JMZ), Jazz Pharmaceuticals (JMZ), Department of Veterans Affairs Sierra-Pacific Mental Illness Research, Education, and Clinical Center (JMZ), Howard Hughes Medical Institute (EM), Swiss National Science Foundation (HPL), and NIH-NS23724 (EM).

\section{References}

1. Adamantidis AR, Zhang F, Aravanis AM, Deisseroth K, de Lecea L. Neural substrates of awakening probed with optogenetic control of hypocretin neurons. Nature 2007;450:420-424. [PubMed: 17943086]

2. Beardsley PM, Balster RL, Harris LS. Evaluation of the discriminative stimulus and reinforcing effects of gammahydroxybutyrate (GHB). Psychopharmacol 1996;127:315-322.

3. Béracochéa D, Liscia P, Tronche C, Chauveau F, Jouanin J-C, Piérard C. Stress modulation of the memory retrograde-enhancing effects of the awakening drug modafinil in mice. Psychopharmacol 2008;196:1-13.

4. Berridge CW, España RA. Hypocretins: waking, arousal, or action? Neuron 2005;46:696-698. [PubMed: 15924855]

5. Brun J, Chamba G, Khalfallah Y, Girard P, Boissy I, Bastuji H, Sassolas G, Claustrat B. Effect of modafinil on plasma melatonin, cortisol and growth hormone rhythms, rectal temperature and performance in healthy subjects during a 36 h sleep deprivation. J Sleep Res 1998;7:105-114. [PubMed: 9682182]

6. Carai MA, Lobina C, Maccioni P, Cabras C, Colombo G, Gessa GL. Gamma-aminobutyric acidB (GABAB)-receptor mediation of different in vivo effects of gamma-butyrolactone. J Pharmacol Sci 2008;106:199-207. [PubMed: 18270475]

7. Castelli MP, Mocci I, Langlois X, Gommeren W, Luyten WHML, Leysen JE, Gessa GL. Quantitative autoradiographic distribution of $\gamma$-hydroxybutyric acid binding sites in human and monkey brain. Mol Brain Res 2000;78:91-99. [PubMed: 10891588]

8. Chemelli RM, Willie JT, Sinton CM, Elmquist JK, Scammell T, Lee C, Richardson JA, Williams SC, Xiong Y, Kisanuki Y, Fitch TE, Nakazato M, Hammer RE, Saper CB, Yanagisawa M. Narcolepsy in 
orexin knockout mice: molecular genetics of sleep regulation. Cell 1999;98:437-451. [PubMed: 10481909]

9. Crunelli V, Emri Z, Leresche N. Unravelling the brain targets of $\gamma$-hydroxybutyric acid. Curr Opinon Pharmacol 2006;6:44-52.

10. Deboer T, Overeem S, Visser NAH, Duindam H, Frölich M, Lammers GJ, Meijer JH. Convergence of circadian and sleep regulatory mechanisms on hypocretin-1. Neurosci 2004;129:727-732.

11. Doherty JD, Hattox SE, Snead OC, Roth RH. Identification of endogenous $\gamma$-hydroxybutyrate in human and bovine brain and its regional distribution in human, guinea pig and rhesus monkey brain. J Pharmacol Exp Therapeutics 1978;207:130-139.

12. Hagan JJ, Leslie RA, Patel S, Evans ML, Wattam TA, Holmes S, Benham CD, Taylor SG, Routledge C, Hemmati P, Munton RP, Ashmeade TE, Shah AS, Hatcher JP, Hatcher PD, Jones DNC, Smith MI, Piper DC, Hunter AJ, Porter RA, Upton N. Orexin A activates locus coeruleus cell firing and increases arousal in the rat. Proc Natl Acad Sci USA 1999;96:10911-10916. [PubMed: 10485925]

13. Hermant J-F, Rambert FA, Duteil J. Awakening properties of modafinil: effect on nocturnal activity in monkeys (Macaca mulatta) after acute and repeated administration. Psychopharmacol 1991;103:28-32.

14. Ivanenko A, Tauman R, Gozal D. Modafinil in the treatment of excessive daytime sleepiness in children. Sleep Med 2003;4:579-582. [PubMed: 14607353]

15. Lagarde D, Milhaud C. Electroencephalographic effects of modafinil, an alpha-1-adrenergic psychostimulant, on the sleep of rhesus monkeys. Sleep 1990;13:441-448. [PubMed: 1981102]

16. Lee MG, Hassani OK, Jones BE. Discharge of identified orexin/hypocretin neurons across the sleepwaking cycle. J Neurosci 2005;25:6716-6720. [PubMed: 16014733]

17. Mignot E, Lammers GJ, Ripley B, Okun M, Nevsimalova S, Overeem S, Vankova J, Black J, Harsh J, Bassetti C, Schrader H, Nishino S. The role of cerebrospinal fluid hypocretin measurement in the diagnosis of narcolepsy and other hypersomnias. Arch Neurol 2002;59:1553-1562. [PubMed: 12374492]

18. Mileykovskiy BY, Kiyashchenko LI, Siegel JM. Behavioral correlates of activity in identified hypocretin/orexin neurons. Neuron 2005;46:787-798. [PubMed: 15924864]

19. Mosteller RD. Simplified calculation of body-surface area. N Engl J Med 1987;317:1098. [PubMed: 3657876]

20. Nakamura RK, Myslobodsky MS, Coppola R, Johannesen-Conway J, Mirsky AF. Effects of $\gamma$ hydroxybutyrate on the performance of monkeys in a Go/No-go visual discrimination task. Beh Brain Res 1987;26:19-27.

21. Piérard C, Liscia P, Valleau M, Drouet I, Chauveau F, Huart B, Bonneau D, Jouanin J-C, Beaumont M, Béracochéa D. Modafinil-induced modulation of working memory and plasma corticosterone in chronically-stressed mice. Pharmacol Biochem Behavior 2006;83:1-8.

22. Qu WM, Huang ZL, Xu XH, Matsumoto N, Urade Y. Dopaminergic D1 and D2 receptors are essential for the arousal effect of modafinil. J Neurosci 2008;28:8462-8469. [PubMed: 18716204]

23. Rao Y, Liu Z-W, Borok E, Rabenstein RL, Shanabrough M, Lu M, Picciotto MR, Horvath TL, Gao $\mathrm{X}-\mathrm{B}$. Prolonged wakefulness induces experience-dependent synaptic plasticity in mouse hypocretin/ orexin neurons. J Clin Investigation 2007;117:4022-4033.

24. Salomon RM, Ripley B, Kennedy JS, Johnson B, Zeitzer JM, Nishino S, Mignot E. Diurnal variation of CSF hypocretin-1 (orexin-A) levels in control and depressed subjects. Biol Psychiatry 2003;54:96104. [PubMed: 12873798]

25. Scammell TE, Estabrooke IV, McCarthy MT, Chemelli RM, Yanagisawa M, Miller MS, Saper CB. Hypothalamic arousal regions are activated during modafinil-induced wakefulness. J Neurosci 2000;20:8620-8628. [PubMed: 11069971]

26. Shelton J, Nishino S, Vaught J, Dement WC, Mignot E. Comparative effects of modafinil and amphetamine on daytime sleepiness and cataplexy of narcoleptic dogs. Sleep 1995;18:817-826. [PubMed: 8746387]

27. Snead OC III. Gamma hydroxybutyrate in the monkey. I. Electroencephalographic, behavioral, and pharmacokinetic studies. Neurol 1978;28:636-642.

28. Tononi G, Cirelli C. Staying awake puts pressure on brain arousal systems. J Clin Investigation 2007; $117: 3648-3650$. 
29. van Vliet SA, Jongsma MJ, Vanwersch RA, Olivier B, Philippens IH. Efficacy of caffeine and modafinil in counteracting sleep deprivation in the marmoset monkey. Psychopharmacol (Berl) 2008;197:59-66.

30. Willie JT, Renthal W, Chemelli RM, Miller MS, Scammell TE, Yanagisawa M, Sinton CM. Modafinil more effectively induces wakefulness in orexin-null mice than in wild-type littermates. Neurosci 2005; 130:983-995.

31. Wisor JP, Eriksson KS. Dopaminergic-adrenergic interactions in the wake promoting mechanism of modafinil. Neurosci 2005;132:1027-1034.

32. Wisor JP, Nishino S, Sora I, Uhl GH, Mignot E, Edgar DM. Dopaminergic role in stimulant-induced wakefulness. J Neurosci 2001;21:1787-1794. [PubMed: 11222668]

33. Woolverton WL, Rowlett JK, Winger G, Woods JH, Gerak LR, France CP. Evaluation of the reinforcing and discriminative stimulus effects of $\gamma$-hydroxybutyrate in rhesus monkeys. Drug Alcohol Dependence 1999;54:137-143.

34. Wu M-F, John J, Maidment N, Lam HA, Siegel JM. Hypocretin release in normal and narcoleptic dogs after food and sleep deprivation, eating, and movement. Am J Physiol 2002;283:R1079-R1086.

35. Yoshida Y, Fujiki N, Nakajima T, Ripley B, Matsumura H, Yoneda H, Mignot E, Nishino S. Fluctuation of extracellular hypocretin-1 (orexin A) levels in the rat in relation to the light-dark cycle and sleep-wake activities. Eur J Neurosci 2001;14:1075-1081. [PubMed: 11683899]

36. Yoshimichi G, Yoshimatsu H, Masaki T, Sakata T. Orexin-A regulates body independent components to hypocretin-1 (orexin A) regulation in sleep-wake consolidating monkeys. J Physiol 2001;557:1045-1053.

37. Zeitzer JM, Buckmaster CL, Lyons DM, Mignot E. Locomotor-dependent and -temperature in coordination with arousal status. Exp Biol Med 2004;226:468-476.

38. Zeitzer JM, Buckmaster CL, Lyons DM, Mignot E. Effects of increasing length of wakefulness on hypocretin-1 in the wake-consolidating squirrel monkey. Am J Physiol 2007;293:R1736-R1742.

39. Zeitzer JM, Buckmaster CL, Parker KJ, Hauck CM, Lyons DM, Mignot E. Circadian and homeostatic regulation of hypocretin in a primate model: implications for the consolidation of wakefulness. $\mathrm{J}$ Neurosci 2003;23:3555-3560. [PubMed: 12716965]

40. Zeitzer, JM.; Mignot, E. Role of hypocretin/orexin in the neurobiology of sleep and alertness. In: Bassetti, C.; Billiard, M.; Mignot, E., editors. Narcolepsy and Hypersomnia. New York: Informa Healthcare; 2006. p. 359-374.

41. Zhang S, Zeitzer JM, Yoshida Y, Wisor JP, Nishino S, Edgar DM, Mignot E. Lesions of the suprachiasmatic nucleus eliminate the daily rhythm of hypocretin-1 release. Sleep 2004;27:619-627. [PubMed: 15282996] 


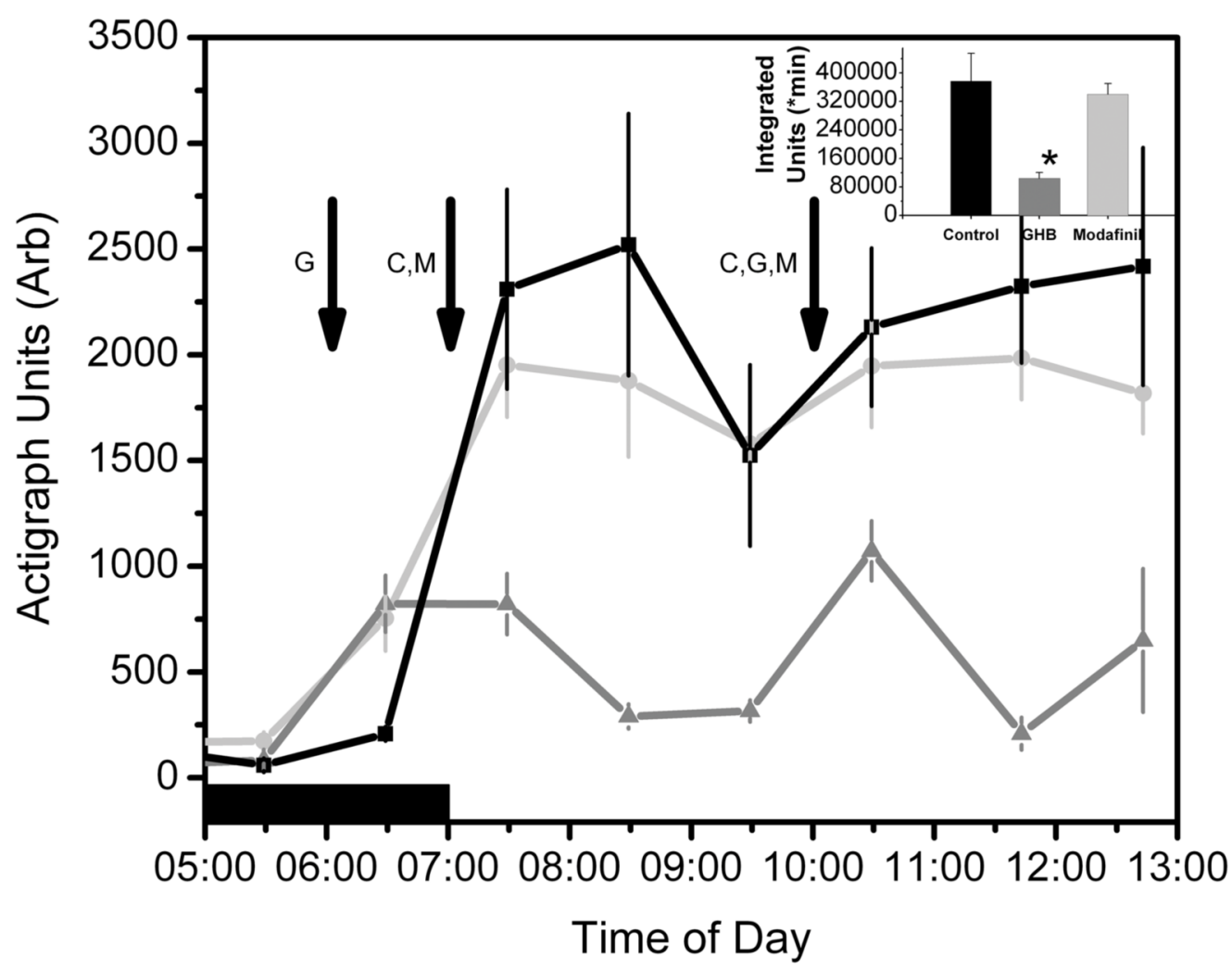

Figure 1.

Average movement from 05:00 until 13:00 in animals exposed to placebo (black), modafinil (light grey), or GHB (dark grey). Data were averaged over an hour within and then between monkeys and are presented as mean \pm SEM. The time of darkness is illustrated by a dark bar above the $\mathrm{x}$-axis. The time of drug/placebo administration is indicated by arrows. Integrated actigraphy data $(07: 00-13: 00)$ is presented in the inset. Significance difference $(\mathrm{p}<0.05)$ from control is indicated by an asterisk. Arb $=$ arbitrary units. 


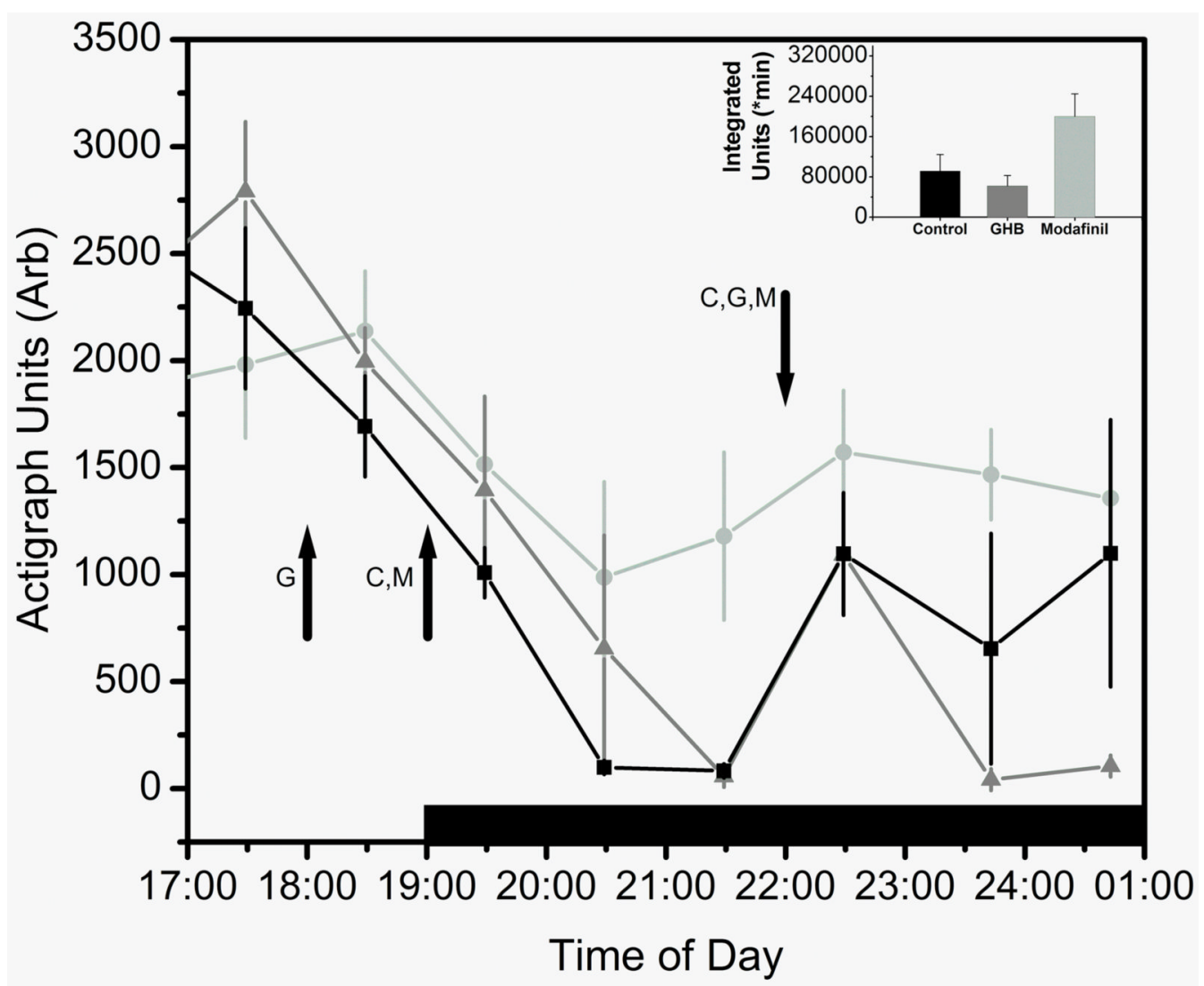

Figure 2.

Average movement from 17:00 until 01:00 in animals exposed to placebo (black), modafinil (light grey), or GHB (dark grey). Legend as for Figure 1 except the inset shows actigraphy data integrated from 20:00 to 01:00. The time of drug/placebo administration is indicated by arrows ( $\mathrm{G}$ for $\mathrm{GHB}, \mathrm{M}$ for modafinil, $\mathrm{C}$ for control). 


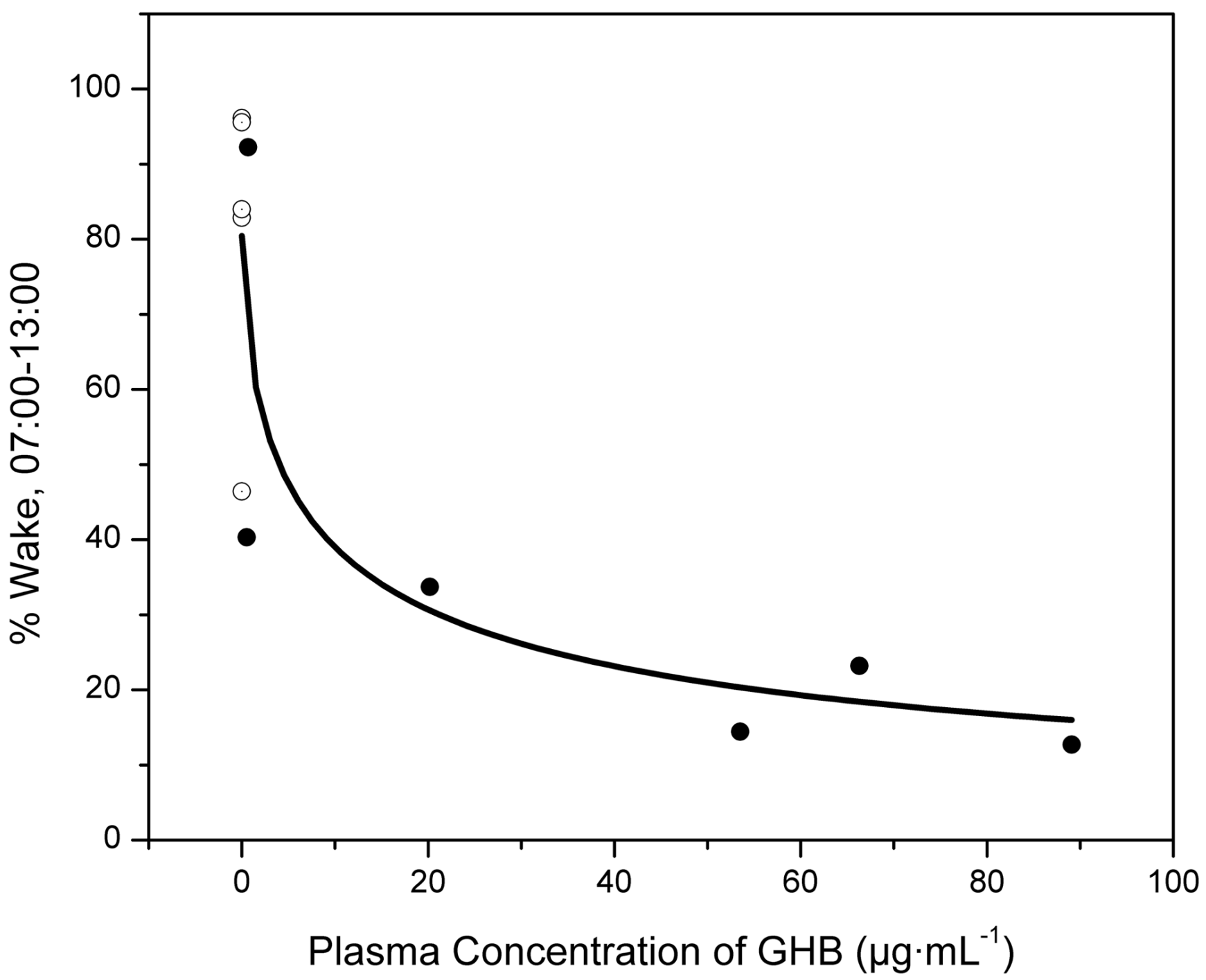

Figure 3.

The amount of wakefulness present between 07:00 and 13:00 after administration of the same injected dose of GHB $(\bullet)$ or a placebo injection ( $(0)$ follows a logistic function $\left[\mathrm{y}=\mathrm{A}_{2}+\right.$ $\left.\left(\mathrm{A}_{1}-\mathrm{A}_{2}\right) \cdot\left(1+\left(\mathrm{x} / \mathrm{x}_{0}\right)^{\mathrm{p}}\right)^{-1}\right]$. 

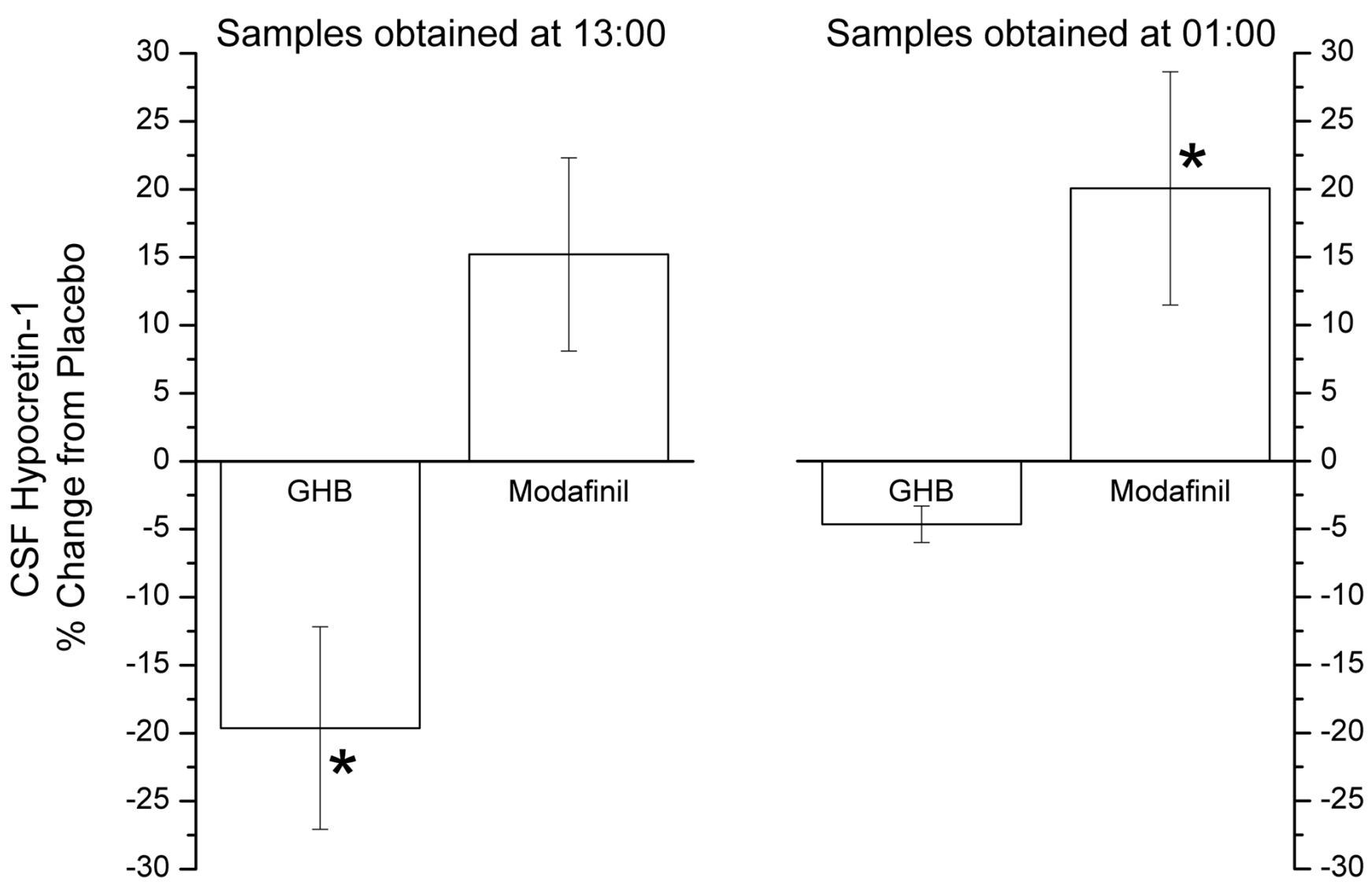

Figure 4.

CSF hypocretin-1 concentration change scores from placebo treatment at 13:00 (left panel) and 01:00 (right panel) after administration of GHB or modafinil. Hypocretin-1 is significantly lower after daytime administration of GHB, when there is an increase in sleep, and is significantly elevated after nighttime modafinil administration, when there is an increase in wakefulness. Hypocretin-1 is increased at 13:00 after modafinil treatment, but this change did not reach statistical significance $(p=0.18)$. Data are plotted as average \pm SD percent change in hypocretin-1 concentrations in the drug conditions, as compared to placebo. Percentage data were $\log$ transformed before averaging and re-transformed following averaging. 

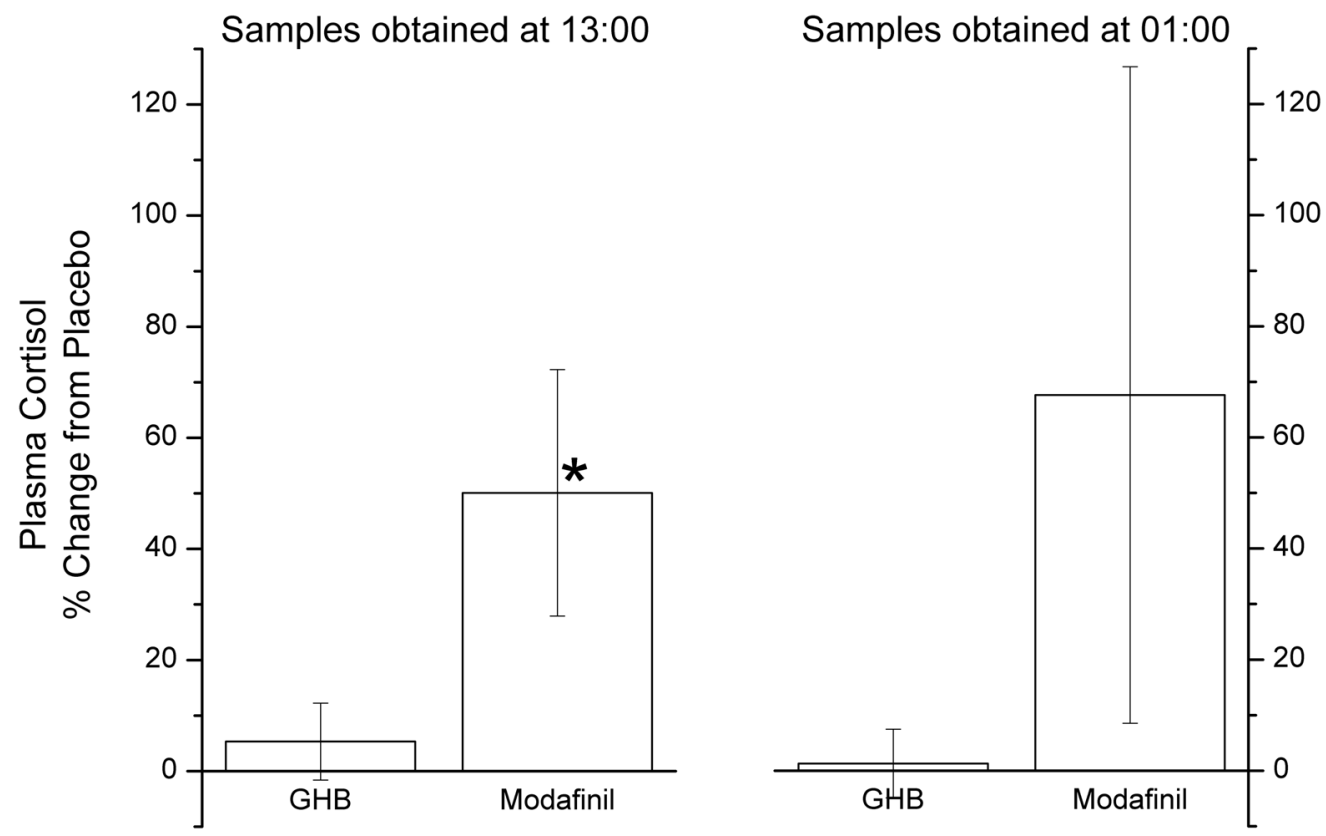

Figure 5.

Plasma cortisol concentration change scores from placebo treatment at 13:00 (left panel) and 01:00 (right panel) after administration of GHB or modafinil. Cortisol was significantly elevated after daytime administration of modafinil, but the increase in cortisol after nighttime administration did not reach statistical significance $(p=0.25)$. There was no significant effect of GHB on cortisol. Data are plotted as average \pm SD percent change in cortisol concentrations in the drug conditions, as compared to placebo. Percentage data were log transformed before averaging and re-transformed following averaging. 